\title{
Opportunistic Routing for Multi-flow Video Dissemination over Flying Ad-Hoc Networks
}

\author{
Denis Rosário*, $^{\dagger}$, Zhongliang Zhao ${ }^{\dagger}$, Torsten Braun ${ }^{\dagger}$, Eduardo Cerqueira*, ${ }^{*}$, Aldri Santos ${ }^{\S}$, and Islam Alyafawi ${ }^{\dagger}$ \\ * Faculty of Computer Engineering and Telecommunication, Federal University of Pará, Belém - Brazil \\ $\dagger$ Institute of Computer Science and Applied Mathematics, University of Bern, Bern - Switzerland \\ $\ddagger$ Computer Science Department, University of California Los Angeles, Los Angeles - USA \\ $\S$ Department of Informatics, Federal University of Paraná, Curitiba - Brazil \\ Email: \{rosario, zhao,braun\}@iam.unibe.ch, cerqueira@ufpa.br, aldri@inf.ufpr.br, alyafawi@iam.unibe.ch
}

\begin{abstract}
A reliable and robust routing service for Flying AdHoc Networks (FANETs) must be able to adapt to topology changes. User experience on watching live video sequences must also be satisfactory even in scenarios with buffer overflow and high packet loss ratio. In this paper, we introduce a Cross-layer Link quality and Geographical-aware beaconless opportunistic routing protocol (XLinGO). It enhances the transmission of simultaneous multiple video flows over FANETs by creating and keeping reliable persistent multi-hop routes. XLinGO considers a set of cross-layer and human-related information for routing decisions, as performance metrics and Quality of Experience (QoE). Performance evaluation shows that XLinGO achieves multimedia dissemination with QoE support and robustness in a multi-hop, multi-flow, and mobile network environments.

Index Terms-Multiple video flows, OR, QoE, and Robustness.
\end{abstract}

\section{INTRODUCTION}

Multi-flow video transmissions over Flying Ad-Hoc Networks (FANETs) enable a large class of multimedia applications, such as natural disaster recovery [1]. However, transmission of video flows over FANETs is a hard task due to topology changes, which might have impact on both network performance and video quality. Moreover, multimedia transmission usually involves multiple nodes transmitting multiple video flows simultaneously [2], leading to a higher degree of buffer overflow and packet loss ratio.

Several routing protocols have been proposed to meet the requirement of delivering video flows with robustness and Quality of Experience (QoE) support over FANET scenarios. Those protocols are based on flat, hierarchical, or geographical approaches, and rely on end-to-end routes to forward packets $[3,4]$. However, end-to-end routes might be subject to frequent interruptions or may not exist at all times. In this context, beaconless Opportunistic Routing (OR) protocols enable packet transmission even in case of continuous topology changes [5]. In addition, the routing service should detect and recover from route failures, enabling a smoother operation. However, crosslayer optimization in beaconless OR protocols taking into account information from network and link layers, as well as user's experience is still an open issue.

To the best of our knowledge, most existing beaconless OR protocols [6-8] do not establish persistent multi-hop routes with robustness and reliability, and also they do not consider QoE requirements for scenarios with multi-flow video transmissions and mobile nodes. Those that attend to establish reliable persistent multi-hop routes, such as LinGO [9], do not enable reliable multimedia transmission with multi-flow video transmissions. LinGO combines link quality, geographical information, and energy for routing decisions, as well as considers dynamic topologies. But, it deals only with node failures and channel quality variations. Moreover, those protocols [6-9] do not prevent the selection of forwarding nodes with heavy traffic load or low residual energy, which causes route failures, queue congestion, packet loss, delay, and jitter. Finally, they rely on periodic route reconstruction, introducing a long time to detect and respond to topology changes, which reduces system robustness and increases packet loss ratio. These factors preclude the ability to achieve multimedia transmission with reliability, robustness, and QoE assurance in scenarios with multiple video flows and mobile nodes.

To address the above issues, this paper introduces the Crosslayer Link quality and Geographical-aware beaconless OR protocol (XLinGO). The main contribution of this paper relies on the combination of a set of cross-layer and human-related parameters for routing decisions, namely packet delivery ratio, QoE, queue length, link quality, geographical location, and residual energy. It also considers a recover mechanism to deal with route failures, providing smoother operation in harsh environments and mobile networks. Based on simulation results, we conclude that XLinGO provides multimedia transmission with robustness and QoE support in scenarios with multiple video flows and mobile nodes.

The remainder of this paper is structured as follows. Section II outlines existing OR protocols and their main drawbacks. Section III introduces the network model used to describe XLinGO. Section IV describes XLinGO. Section V discusses simulation results. Section VI concludes this paper.

\section{RELATED WORK}

Ancillotti et al. [3] developed a queue network model to predict the residual capacity of end-to-end paths and to identify network bottlenecks in wireless networks. Xu et al. [4] introduced a routing protocol that searches suitable paths for video streams, by selecting paths with sufficient node capacities and reduced interference. However, both proposals 
$[3,4]$ consider end-to-end routes, and do not evaluate the impact of multimedia dissemination based on user perspective.

Heissenbüttel et al. [6] introduced the concept of Dynamic Forwarding Delay (DFD) as a forwarding decision in the BeaconLess Routing protocol (BLR). The source node broadcasts a data packet, and before forwarding the received packet, possible relays within a forwarding area compute a DFD value based only on location information. Thus, the node closest to the destination generates the shortest DFD and forwards the packet first. Neighbour nodes recognize the occurrence of relaying, and cancel their scheduled transmission for the same packet that they overhear. Sanchez et al. [7] proposed the Beaconless On-demand geographic routing Strategy (BOSS), which extends BLR by introducing a different forwarding area and applying a three-way handshake mechanism. BOSS assumes a full data payload for the Request to Send (RTS) message size, since selecting a forwarder using small control messages may lead to the choice of a forwarder unable to receive larger data packets. Al-Otaibi et al. [8] proposed a Multipath Routeless Routing protocol (MRR), which defines a forwarding area as a rectangle and combines multiple metrics to compute the DFD. In MRR, when a given node receives a packet with weaker signals, it forwards the packet first.

However, BLR and BOSS rely on a single metric to compute the DFD. For instance, computing DFD based only on distance increases packet loss ratio and reduces video quality, since the most distant node might suffer from bad connectivity. BOSS includes overhead and delay for the three-way handshake mechanism. MRR selects a forwarding node that received the packet with weaker signals, reducing the system reliability as well as the video quality level. Finally, BLR, MRR, and BOSS rely on a periodic route reconstruction to detect topology changes. But, when one of the forwarding nodes from a given route is no longer available to forward packets, a burst of packets might be lost until the protocol re-establishes the route. These protocols also do not preclude the selection of forwarding nodes with heavy traffic load or low residual energy.

\section{System MODEL}

Multimedia dissemination over FANETs provides visual information, as soon as the standard fixed network infrastructure is unavailable due to a natural disaster. Hence, multimedia content plays an important role in enabling humans in the control center to take appropriate actions to explore a hazardous area, which rescuers are unable to reach easily and quickly.

\section{A. Network Model}

We consider a network composed of $n$ mobile nodes deployed in the monitored area. Each node has an individual identity $(i \in[1, n])$, and these nodes are represented in a dynamic graph $G(V, E)$, where vertices $V=\left\{v_{1}, v_{2}, \ldots, v_{n}\right\}$ represent a finite set of nodes, and edges $E=\left\{e_{1}, e_{2}, \ldots, e_{m}\right\}$ build a finite set of wireless links between the mobile nodes $\left(v_{i}\right)$. We define $\mathrm{N}\left(v_{i}\right) \subset V$ as a subset of neighbours within the radio range of a given node $v_{i}$. We assume a network scenario composed of one static Destination Node $(D N) \subset$ $V$ equipped with a radio transceiver, an image decoder, and unlimited energy supply. On the other hand, each mobile node $v_{i}$ is equipped with a camera, an image encoder, a radio transceiver, and a limited energy supply. For convenience of notation, we denote $S N \subset V$ (Source Node) as the node $v_{i}$ responsible for capturing video flows and transmitting them to the $D N$ in a multi-hop fashion.

Each link $e_{j}, j=1, \ldots, m$ has a weight value associated $\left(w\left(e_{j}\right)\right)$, which is equivalent to the link quality perceived by node $v_{i+1}$ for a packet received from node $v_{i}$. For instance, the physical layer of the CC2420 radio chip provides the Received Signal Strength Indicator (RSSI), Signal to Noise Ratio (SNR), and Link Quality Indicator (LQI), which are usually used to estimate the link quality of each received packet [10]. Each node $v_{i}$ has a queue $(Q)$ with a maximum queue capacity $\left(Q_{\max }\right)$ and current queue length $\left(Q_{\text {length }}\right)$. The queue policy schedules the packet transmission by using the First In First Out (FIFO) algorithm, and drops packets using the Drop Tail algorithm in case of buffer overflow, as required in FANETs.

Each node $v_{i}$ has a battery with initial power $\left(P_{0}\right)$. It requires power $\left(P_{t x}\right)$ to transmit a packet, $\left(P_{r x}\right)$ to receive a packet, and $\left(P_{v}\right)$ to move at a certain speed $\left(s_{v i}\right)$. It moves to a certain direction $(\overrightarrow{d i r})$ with the speed ranging between a minimum $\left(s_{\min }\right)$ and a maximum $\left(s_{\max }\right)$ limit. Each node $v_{i}$ is aware of its own location by means of GPS, or any other positioning service. The $D N$ location is known a priori by each node $v_{i}$, since we assume a static $D N$.

\section{B. Problem Statement}

In this paper, we find a subset of reliable forwarders $F$ to establish a persistent route $P_{S N, D N}=\left\{S N, F_{1}, \ldots, F_{n}, D N\right\}$ $\subset V$ in order to connect $S N$ to $D N$. In particular, the subset of optimal $F$ must provide packet delivery guarantees, and thus enable video transmission with a low packet loss rate. We prevent the selection of a node $v_{i}$ with heavy traffic load or low residual energy from being selected as $F$. In this way, we provide load balancing, energy-efficiency, and minimize the impact of buffer overflows. Finally, we determine whether one of the $F_{i}$ from a given persistent route $P_{S N, D N}$ might be no longer available to forward packets. Hence, we provide robust and reliable multimedia transmission over FANET scenarios.

\section{The XLingo Protocol}

In this section, we describe XLinGO, which combines multiple metrics to build reliable persistent routes and considers a mechanism to recover from route failures.

\section{A. Contention-based Forwarding Mode}

Whenever a given $S N$ wants to send a video flow, it triggers the contention-based forwarding mode by broadcasting the video packet to its neighbours $\mathrm{N}(S N)$. Before the $S N$ transmits a video packet, it must determine its own location $\left(x_{S N}, y_{S N}\right)$ and include this information in the packet header.

$S N$ 's neighbours $\mathrm{N}(S N)$ compete to forward the received packets in a completely distributed manner, and XLinGO 
attempts to ensure that only one node forwards the packet. This is accomplished by $\mathrm{N}(S N)$ computing the DFD, the required energy to move and transmit packets, and also by restricting the area in which nodes are allowed to forward the packet (called forwarding area).

More specifically, as soon as $\mathrm{N}(S N)$ receive a packet, they can find out the $S N$ location by analysing the packet header. $\mathrm{N}(S N)$ are aware of their own locations and $D N$ location. Thus, $\mathrm{N}(S N)$ can classify their forwarding area into: Positive Progress Area (PPA) and Negative Progress Area (NPA). PPA comprises the area, where $\mathrm{N}(S N)$ are closer to $D N$ than $S N$, while NPA comprises the other neighbours, as shown in Figure 1. Each $N(S N)$ located within NPA must drop the received packet, since they are further away from $D N$ than $S N$. On the other hand, nodes within the PPA are considered as possible relay nodes $\left(R N_{i} \in \mathrm{N}(S N)\right)$.

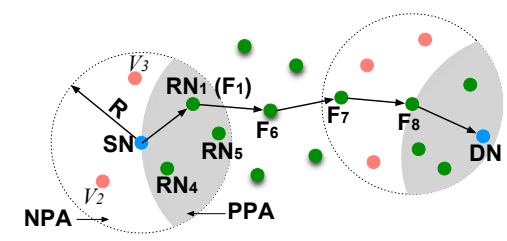

Fig. 1. Forwarding Area and Forwarding Strategy

Battery-powered mobile nodes should consider Residual Energy $(R E)$ for forwarding decisions with the aims to prevent the selection of a forwarding node with low $R E$. This is accomplished by each node of $\mathrm{N}(S N)$ within the PPA, computing the Energy threshold $\left(E_{t h}\right)$, to check if the $R E$ falls below the $E_{t h} . E_{t h}$ indicates the total amount of energy required to transmit packets $\left(E_{t x}\right)$ and to move $\left(E_{v}\right)$, where $R N_{i}$ require $\left(E_{t x}=k^{\prime} \times P_{t x}\right)$ to transmit a given number of $k^{\prime}$ packets, and $\left(E_{v}=s \times P_{v}\right)$ to move at a certain speed $s$. Hence, a given $R N_{i}$ only becomes a candidate, if it has enough $R E$ to forward subsequent video packets, and to move back to the control centre for battery replacement.

Instead of immediately forwarding the received packet, each $R N_{i}$ must compute the DFD value in the interval [0, $D F D_{M a x}$ ], start a timer according to the DFD value, and wait for the expiration of this timer to forward the received packet. In this way, the $R N_{i}$ that generates the smallest DFD value, replaces the $S N$ location with its own locations in the packet header, and forwards the packet first, becoming forwarder node $(F \in \mathrm{N}(S N))$. Each $R N_{i}$ must cancel its scheduled transmission, and delete the buffered packet by overhearing a retransmission coming from $F$. At the same time, XLinGO uses the transmitted packet for passive acknowledgement, and thus the $S N$ knows which $R N_{i}$ has been selected as $F$, in order to unicast the subsequent packets. The algorithm continues until the packet reaches $D N$, which sends an explicit acknowledgement. Hence, XLinGO establishes a reliable persistent route $P_{S N, D N}$, connecting $S N$ and $D N$ via multiple $F$.

The hidden terminal problem might appear, and $S N$ may be unable to overhear the packet retransmission from $F$. If this occurs, it prevents $S N$ from establishing a route. Route creation may also fail if there is not any $R N_{i}$ inside the PPA. Hence, $S N$ must repeat the contention-based forwarding mode until it establishes the $P_{S N, D N}$, when the $S N$ does not overhear a packet retransmission by any $R N_{i}$ within $D F D_{\text {Max }}$.

\section{B. Metrics for the Contention-based Forwarding Mode}

Each $R N_{i}$ computes the DFD based on Eq. (1), which includes coefficients $(\alpha, \beta$, and $\gamma)$ to give importance to each metric, and the sum of the coefficients is equal to 1 . In contrast to our previous work [9], in this paper we define each metric by means of exponential distribution, reducing the number of responses and leading to a better feedback suppression [6].

$$
\begin{array}{r}
\mathrm{DFD}=D F D_{\operatorname{Max}} \times(\alpha \times \text { linkQuality }+\beta \times \text { progress }+ \\
\gamma \times \text { queueLength })
\end{array}
$$

1) Link Quality: XLinGO considers link quality as part of the DFD function, which ensures that the selected forwarding node $F$ provides multimedia transmission with packet delivery guarantees. In particular, each link $e_{j}$ has a weight value $w\left(e_{j}\right)$ associated, which represents a single value for the link quality computed at the received side, such as provided by RSSI, SNR, or LQI. Our previous work [9] classifies links according to Packet Reception Ratio (PRR). In this paper, we define $w\left(e_{j}\right)$ thresholds based on the video quality level that a given link provides. In this way, we provide QoE-awareness.

We defined two $w\left(e_{j}\right)$ thresholds, i.e., $w\left(e_{j}\right)_{b a d}$ and $w\left(e_{j}\right)_{\text {good }}$, according to calibration experiments. Such experiments established a rich set of links exhibiting different qualities, independently of any external factor, such as collisions and routing. Hence, we deployed 40 nodes with different distances and directions from the $D N$, since distance and direction directly affect the link quality. Nodes were placed in a circle around the $D N$, each circle had 4 nodes, and the distance between two consecutive circles was equal to 1 meter. Each node had an exclusive time slot defined according to a Time Division Multiple Access (TDMA) scheme, to transmit a video to the $D N$. Figure 2 presents the Structural Similarity (SSIM) as a function of the $w\left(e_{j}\right)$, where it is possible analyse the video quality with the link quality $w\left(e_{j}\right)$.

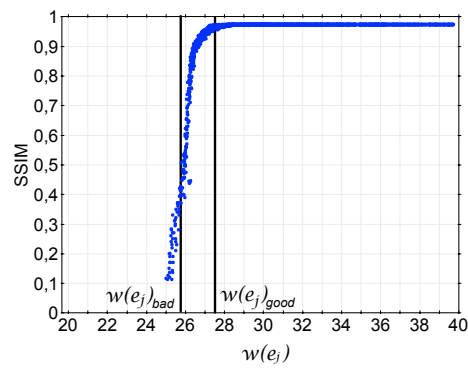

Fig. 2. Link Quality vs. Video Quality

Regarding XLinGO's operation, a given $R N_{i}$ must drop the received packet, as soon as it receives a packet with link quality $w\left(e_{j}\right)$ lower than $w\left(e_{j}\right)_{b a d}$. This is because our experiments showed that such link provides video transmission with a poor quality level, i.e., SSIM lower than 0.5. LinkQuality 
adds 0 to $D F D$ function, when a given $R N_{i}$ receives a packet with $w\left(e_{j}\right)$ higher than $w\left(e_{j}\right)_{\text {good }}$. This is because this node provides multimedia dissemination with QoE assurance, and XLinGO must increase the probability of this node forwards the packet faster. Finally, for $w\left(e_{j}\right)_{j}$ between $w\left(e_{j}\right)_{b a d}$ and $w\left(e_{j}\right)_{\text {good }}$, the LinkQuality value is computed based on Eq. (2) in the interval from 0 to 1 . Hence, when a given $R N_{i}$ receives a packet from $S N$ with low $w\left(e_{j}\right)$, it computes a higher LinkQuality value to reduce its likelihood of become $F$. This is because a given $R N_{i}$ with low $w\left(e_{j}\right)$ cannot provide multimedia transmission with QoE assurance.

$$
\text { linkQuality }=\frac{1}{1+e^{-c\left(w\left(e_{j}\right)-x\right)}}
$$

We employed the sigmoid function for the exponential distribution, which requires parameter $x$ to determine the center of the sigmoid output, and $c$ to regulate the slope or "growth rate" of the sigmoid during its rising portion. We used a negative value for the parameter $c$, to enable large values of $w\left(e_{j}\right)$ to generate output closer to 0 .

2) Progress: Through the progress metric, we attempt to minimize the number of hops, since longer routes reduce the packet delivery ratio. For this reason, we prefer to select the $F$ closer to the $D N$. Hence, a given $R N_{i}$ with a larger geographical advance towards $D N$ generates a smaller progress value. The progress value is computed according to:

$$
\text { progress }=\frac{1}{1+e^{\left(-c\left(P\left(R N_{i}, D N\right)-R\right)\right)}}
$$

We denote $R$ as the radio range, which should be the center of the sigmoid output. $P\left(R N_{i}, D N\right)$ is equals to the sum of $P_{1}\left(R N_{i}\right)$ and $P_{2}\left(R N_{i}\right)$. We define $P_{1}\left(R N_{i}\right)$ as a projection of the distance travelled from $S N$ to any $R N_{i}$, onto the line from $S N$ to $D N$. On the other hand, the projection of line $R N_{i}$ $R N_{i}^{\prime}$ onto line $S N-D N$ defines $P_{2}\left(R N_{i}\right)$. Thus, $P\left(R N_{i}, D N\right)$ means the geographical advance of a given $R N_{i}$ towards $D N$, as shown in Figure 3. Based on the proposed progress function, a given $R N_{i}$ with less progress adds a larger contribution to the DFD value than a node with more progress.

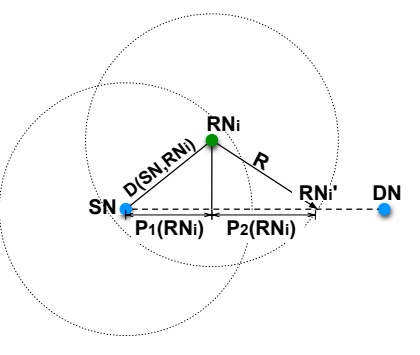

Fig. 3. Progress Definition

3) Queue Length: In a scenario with multiple video flows, buffer overflow in intermediate $F$ might occur. XLinGO considers queue length in order to allow the establishment of a persistent route that avoids the selection of $F$ with heavy traffic load. Hence, it prevents buffer overflow, minimizes packet loss, delay, and also provides load balancing.
To compute the queueLength, each $R N_{i}$ must consider the number of video flows ( $n$ Flow) that it will forward, if it wins the competition to forward the received packet of a new video flow. It also considers the packets per second required for

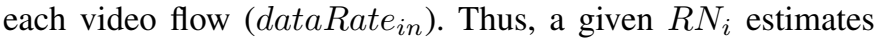
the incoming data rate by taking into account all video flows $\left(n F l o w \times\right.$ dataRate $\left._{i n}\right)$. In addition, each $R N_{i}$ knows the packets per second that it is transmitting ( rate $\left._{\text {out }}\right)$. Hence, a given $R N_{i}$ is able to predict the queue size $\left(Q_{t+1}\right)$ according to Eq. (4), if it wins the competition to forward packets.

$$
Q_{t+1}=\text { rate }_{\text {out }}-\left(\text { nFlow } \times \text { dataRate }_{\text {in }}\right)
$$

Then, each $R N_{i}$ must compute queueLength according to Eq. (5). The proposed function adds a small value to the DFD function, as long soon as the $Q_{t+1}$ in a given $R N_{i}$ is lower.

$$
\text { queueLength }=\frac{1}{1+e^{\left(-c\left(Q_{t+1}-Q_{\max } / 2\right)\right)}}
$$

\section{Persistent Route Mode}

The transmission of all packets in contention-based forwarding mode causes additional delays and interferences, which reduce the video quality level based on the user experience. Hence, XLinGO avoids the drawbacks of broadcasting transmissions by introducing a persistent route mode. In particular, it establishes a persistent route $P_{S N, D N}$ between any pair of $S N$ and $D N$ via multiple $F$ to transmit subsequent packets by using unicast communication.

The video content should be delivered even in presence of continuous topology changes. In this way, existing beaconless OR protocols rely on periodical route reconstruction to detect topology changes [6-8]. On the other hand, XLinGO relies on a recovery mechanism to detect and respond to route failures, allowing a smoother operation in harsh environments.

XLinGO considers that every node that composes a persistent route $P_{S N, D N}$ should assess whether $P_{S N, D N}$ is still a reliable or valid route to transmit packets. Let us illustrate the behaviour of this mechanism using Figure $4 . F_{2}$ receives a given number of $k$ packets from $F_{1}$. Then, $F_{1}$ must compute PRR and the exponential average for $w\left(e_{j}\right)$ perceived in the last $k$ packets. We used the exponential average, since this gives higher importance to the most recent information. $F_{2}$ should send a reply message to $F_{1}$, and piggyback the $w\left(e_{j}\right)$ exponential average and $\mathrm{PRR}$ for the last $k$ received packets. As result, any node from $P_{S N, D N}$ must return to the contention-based forwarding mode, when it detects that the link quality falls below the link quality threshold $w\left(e_{j}\right)_{b a d}$ or has a lower PRR. This is explained in our experiments, which showed that this link provides video dissemination with a poor video quality level, as it can be seen in Figure 2 .

In addition, any node that composes a path $P_{S N, D N}$ must consider that the persistent route is not valid anymore, as long as it does not receive any reply message from its forwarder node within a certain period of time, i.e., time-out. Hence, it must return to the contention-based forwarding mode to reestablish the persistent route. 


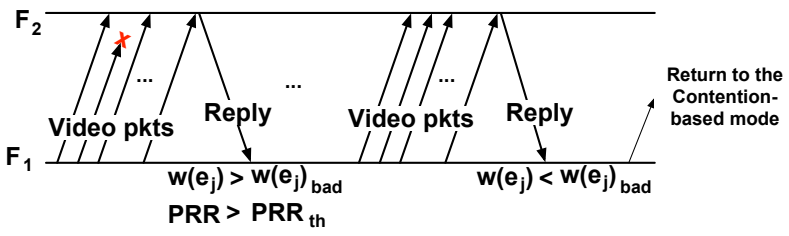

Fig. 4. Time Diagram of the mechanism to recover from route failures

\section{Performance Evaluation}

This section describes the methodology and metrics used to evaluate the quality level of transmitted videos via XLinGO compared to well-know beaconless OR protocols. We evaluated the impact of node mobility on the video quality.

\section{A. Simulation Scenario and Evaluation Metrics}

We used the Mobile MultiMedia Wireless Sensor Network (M3WSN) OMNeT++ framework [11]. The simulations last for 200 seconds (s) and run with the lognormal shadowing path loss model. We set the simulation parameters to allow wireless channel temporal variations, link asymmetry, and irregular radio ranges, as expected in FANETs environment. The results are averaged over 33 simulation runs with different randomly generated seeds to provide a confidence interval of $95 \%$. In our simulations, a network topology is generated with the $D N$ located at location $(50,0)$. The other nodes are moving following the Random Waypoint mobility model over the entire flat terrain of $100 \times 100 \mathrm{~m}$. Nodes are equipped with IEEE 802.11 radio, using a transmission power of $12 \mathrm{dBm}$. Nodes rely on CSMA as a MAC protocol, and the QoE-aware redundancy mechanism [12] to add redundant packets only to priority frames at the application layer.

DFD weights $(\alpha, \beta$, and $\gamma$ ) affect performance of XLinGO. We optimized results showed in [9], and thus XLinGO considers $D F D_{\text {Max }}=100 \mathrm{~ms}, \alpha=0.4, \beta=0.4$, and $\gamma=0.2$. The proposed recovery mechanism considers time - out $=0.3 \mathrm{~s}$ and $P R R_{t h}=30 \%$ to detect topology or channel changes.

In our simulations, $S N$ transmitted the Hall and UAV video sequences downloaded from the YUV video trace library and YouTube. The Hall video sequence has similar characteristics as if a mobile node stopped in a certain area to capture a video. The UAV video sequence has similar motion and complexity levels, compared to a UAV capturing video flows while it is flying. We encoded those videos with H.264 codec at 300 kbps, 30 frames per second, GoP size of 18, and common intermediate format (352 x 288). The decoder uses FrameCopy for error concealment to replace each lost frame with the last received one, since from the user's perspective, the frame losses have less severe impact on the video quality.

QoE metrics overcome the Quality of Service (QoS) scheme limitations to assess the quality level of multimedia applications. Hence, we rely on a well-known objective QoE metric, namely Structural Similarity (SSIM), which ranges from 0 to 1 , and a higher SSIM value means better video quality.

\section{B. Impact of the mechanism to recover from route failures}

We performed a simple simulation scenario to validate the mechanism to recover from route failures. We created the worst-case scenario for the topology changes, where the $S N$ established the persistent route $\left(P_{S N, D N}\right)$ and $10 \%$ of 1-hop neighbours of a $S N$ have individual node failures. We defined three configurations for XLinGO: i) without node failures and without the mechanism to recover from route failures (denoted as XLinGO); ii) with node failures and with periodic route reconstruction, such as performed in LinGO, BLR, MRR, and BOSS (XLinGO - Failure); and iii) with node failures and with the recovery mechanism (XLinGO - Recovery). The original video in the plot represents an errorless video transmission and is used as a benchmark video quality. This is due to video coding and decoding process also introduces impairments in the video quality even in the absence of packet losses. Thus, it helps to see exactly the quality loss due to packet loss.

Figure 5 shows the SSIM for all frames that compose the Hall video sequence for those three XLinGO configurations. XLinGO without node failures keeps the video quality high and constant, i.e., $S S I M$ around 0.94. This is because XLinGO builds a reliable $P_{S N, D N}$, which protects the video frames during link error periods. Hence, XLinGO enables video dissemination with QoE support in scenarios with dynamic topologies caused only by channel quality variations.

Frames $0-17$ have a good quality for XLinGO - Failure and XLinGO - Recovery. This is because $D N$ uses Frame-Copy as error concealment method. Thus, the decoder replaces each lost frame with the last received one, enabling to reconstruct those frames with good quality. XLinGO transmits video frames $18-89$ with bad quality in scenarios with node failures and without a mechanism to recover from route failures (i.e., XLinGO - Failure). The reason for this is that nodes established the $P_{S N, D N}$ and $10 \%$ of network nodes have a node failure. Apart from the topology changes caused by node failures, bursts of packets were lost until the $S N$ re-establishes $P_{S N, D N}$, since one of the nodes from the $P_{S N, D N}$ is no longer available to forward packets. In the worst case, this lasts during the interval for route reconstruction. In our experiments, XLinGO - Failure reconstructs routes every 3 seconds, and this explains the poor video quality for frames $18-89$.

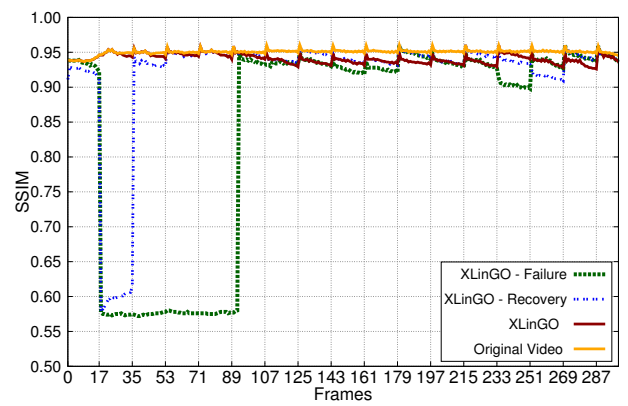

Fig. 5. SSIM for all frames for the Hall video sequence

XLinGO transmitted frames 18 - 34 with poor quality, in scenarios with node failures and a mechanism to recover from 
route failures (i.e., XLinGO - Recovery). This is because the proposed recovery mechanism has a time-out value of $0.5 \mathrm{~s}$, which is the time required to detect that one of the forwarder nodes is no longer available to forward packets. As soon as the time-out expires, the node must return to the contentionbased forwarding mode, and re-establish a reliable $P_{S N, D N}$. We conclude that the proposed recovery mechanism reduces the video quality level less than periodic route reconstruction schemes. This is because the proposed mechanism enables $\mathrm{XLinGO}$ to quickly detect and respond to topology changes, and thus it enables multimedia dissemination with robustness.

\section{Impact of Node Mobility}

In this section, we evaluate the reliability of XLinGO compared to LinGO [9], BLR [6], BOSS [7], and MRR [8] in a scenario composed of mobile nodes and multiple flows. This involves deployment of 40 nodes, where the network has one $D N$, two mobile multimedia source nodes transmitting simultaneous video flows, and 37 possible mobile relay nodes.

Figure 6 shows the video quality level for the videos transmitted via XLinGO, LinGO, BLR, BOSS, and MRR with different node moving speeds. In this way, we can analyse the impact of the moving speed on the final video quality level. For video transmitted through LinGO, BLR, MRR, and BOSS, as soon as the $s_{\max }$ increases, the video quality decreases. This is because forwarders $F$ move out of each other's transmission range, breaking quickly $P_{S N, D N}$, and those protocols rely on periodic route reconstruction, which increases the packet loss ratio in the case of route failures.

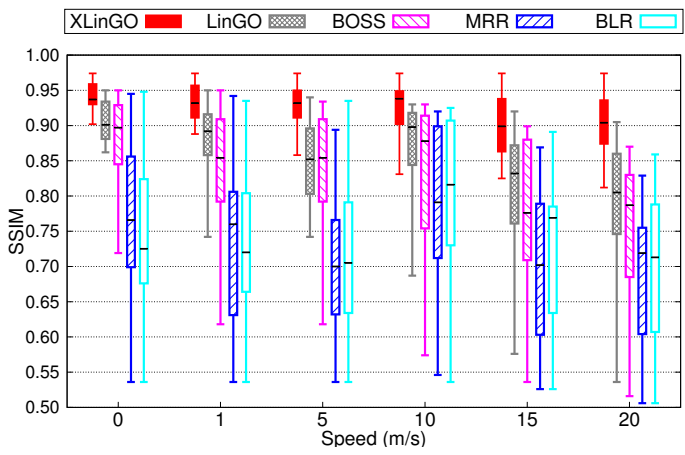

Fig. 6. Video Quality According to Moving Speed

Based on the simulation results, we conclude that XLinGO provides multimedia dissemination with a high quality level compared to LinGO, BLR, MRR, and BOSS regardless of the moving speed. This is because BLR only considers geographical information to compute the DFD, and due to the unreliability of wireless channel, the most distant node might suffer from a bad connection, increasing the packet loss ratio for BLR. In addition, MRR selects a forwarding node that receives a packet with a weak signal, reducing reliability and quality level of videos transmitted via MRR. Videos transmitted via BOSS have a higher quality compared to BLR and MRR, even if BOSS only considers geographical information for routing decision like BLR, since BOSS considers a three-way handshake mechanism to select F. However, videos transmitted via BOSS have a video quality lower than videos transmitted via LinGO and XLinGO. Finally, XLinGO increases video quality compared to LinGO, because XLinGO considers a set of cross-layer and human-related parameters for routing decisions, namely PRR, QoE, queue length, link quality, geographical location, and energy, and also a mechanism to recover from route failure.

\section{CONCLUSION}

This paper introduced XLinGO to provide efficient, robust, and reliable video dissemination together with QoE support in multimedia FANET scenarios. XLinGO takes multiple metrics into account to build reliable multi-hop persistent routes, and also enables the detection and reaction to topology changes. Simulation results highlighted XLinGO's reliability, robustness, and QoE support in face to node mobility and multiple video flows. Based on simulation results, we conclude that XLinGO provided simultaneous multi-flow video dissemination with a greater degree of robustness and QoE support compared to LinGO, BLR, BOSS, and MRR.

\section{ACKNOWLEDGMENTS}

This work is supported by the National Council for Scientific and Technological Development (CNPq), and also PROPESP/FADESP/UFPA.

\section{REFERENCES}

[1] İ. Bekmezci, O. K. Sahingoz, and Ş. Temel, "Flying ad-hoc networks (FANETs): A survey," Ad Hoc Networks, vol. 11, pp. 1254-1270, 2013.

[2] C. Ververidis, J. Riihijarvi, and P. Mahonen, "Evaluation of quality of experience for video streaming over dynamic spectrum access systems," in IEEE International Symposium on a World of Wireless Mobile and Multimedia Networks (WoWMoM). Montreal: IEEE, 2010, pp. 1-8.

[3] E. Ancillotti, R. Bruno, M. Conti, and A. Pinizzotto, "Load-aware routing in mesh networks: Models, algorithms and experimentation," Computer Communications, vol. 34, no. 8, pp. 948-961, 2011.

[4] Y. Xu, J. Deng, M. Nowostawski, and M. Purvis, "Optimized routing for video streaming in multi-hop wireless networks using analytical capacity estimation," Journal of Computer and System Sciences, 2013.

[5] C.-J. Hsu, H.-I. Liu, and W. K. G. Seah, "Survey paper: Opportunistic routing - a review and the challenges ahead," Computer Network, vol. 55, no. 15 , pp. $3592-3603$, oct. 2011.

[6] M. Heissenbüttel, T. Braun, T. Bernoulli, and M. Wälchli, "BLR: beacon-less routing algorithm for mobile ad hoc networks," Computer Communications, vol. 27, no. 11, pp. 1076 -1086, 2004.

[7] J. Sanchez, P. Ruiz, and R. Marin-Perez, "Beacon-less geographic routing made practical: challenges, design guidelines, and protocols," IEEE Communications Magazine, vol. 47, no. 8, pp. 85-91, 2009.

[8] M. Al-Otaibi, H. Soliman, and J. Zheng, "A multipath routeless routing protocol with an efficient location update mechanism," Inter. Journal of Internet Protocol Technology, vol. 6, no. 1/2, pp. 75-82, 2011.

[9] D. Rosário, Z. Zhao, T. Braun, E. Cerqueira, A. Santos, and Z. Li, "Assessment of a robust opportunistic routing for video transmission in dynamic topologies," in Wireless Days conference, Valencia, 2013.

[10] N. Baccour, A. Koubâa, L. Mottola, M. A. Zúñiga, H. Youssef, C. A. Boano, and M. Alves, "Radio link quality estimation in wireless sensor networks: A survey," ACM Transactions on Sensor Networks (TOSN), vol. 8, no. 4, pp. 34:1-34:33, Sep. 2012.

[11] D. Rosário, Z. Zhao, C. Silva, E. Cerqueira, T. Braun, An OMNeT++ Framework to Evaluate Video Transmission in Mobile Wireless Multimedia Sensor Networks, in: Proceedings of the 6th International Workshop on OMNeT++, ICST, Cannes, France, 2013, pp. 277-284.

[12] Z. Zhao, T. Braun, D. Rosário, E. Cerqueira, R. Immich, and M. Curado, "QoE-aware FEC mechanism for intrusion detection in multi-tier WMSNs," in Inter. Workshop on WMSNs, Barcelona, 2012, pp. 697-704. 